\title{
Metachronous Gastrointestinal Stromal Tumor and Acute Leukemia after Liver Transplantation for Cholangiocellular Carcinoma: Is There a Link?
}

\author{
Anna Mrzljak ${ }^{a} \quad$ Iva Košuta $^{a} \quad$ Anita Škrtićb $^{\text {Ika Kardum-Skelin }}{ }^{b}$ \\ Radovan Vrhovac ${ }^{a}$ \\ Departments of ${ }^{a}$ Medicine and ${ }^{b}$ Pathology and Cytology, University Hospital Merkur, \\ School of Medicine, University of Zagreb, Zagreb, Croatia
}

\section{Key Words}

Acute leukemia · Cholangiocellular carcinoma - Gastrointestinal stromal tumor .

Liver transplantation

\begin{abstract}
The synchronous or metachronous coexistence of gastrointestinal stromal tumors (GISTs) with solid and hematologic neoplasms has been addressed in a non-transplant population. However, the association with primary hepatic neoplasms and leukemias is uncommon. Scarce data exist considering association of GISTs and other neoplasms in a transplant population where long-term immunosuppression carries the additional burden of de novo malignancy. We present a case of posttransplant metachronous GIST and acute biphenotypic leukemia in a patient transplanted for intrahepatic cholangiocellular carcinoma, emphasizing the possible link between mechanisms of carcinogenesis and influence of other factors upon their development.
\end{abstract}

\section{Background}

Gastrointestinal stromal tumors (GISTs) are a heterogenous group of mesenchymal tumors of the gastrointestinal tract (GIT) that account for $<1 \%$ of all GIT neoplasms. The synchronous or metachronous coexistence of GISTs with other solid tumors, mainly luminal gastrointestinal and genitourinary neoplasms, reaches an incidence up to $30 \%$ in a non- 
transplant population, wherein the association with primary hepatic neoplasms is uncommon $[1,2]$. The occurrence of GISTs with hematologic neoplasms such as leukemia is considered an uncommon entity, mainly noted in case reports or series; however, recent data suggest an apparent nonrandom association between GISTs and myeloid leukemia [3]. The transplant population carries a risk of malignancy that is 2-4 times higher than in the general population [4]. However, after liver transplantation, de novo GISTs [5, 6] and de novo acute leukemias [7-9] still represent uncommon events that are mainly associated with long-standing immunosuppression.

Herein, we present a case of posttransplant metachronous GIST and acute biphenotypic leukemia after liver transplantation for intrahepatic cholangiocellular carcinoma (ICCC), considering the possible link between mechanisms of carcinogenesis and influence of other factors upon their development.

\section{Case Report}

In 2007, a 53-year-old man underwent liver transplantation with duct-to-duct biliary anastomosis for alcoholic liver cirrhosis. The pathohistology of the explanted liver revealed small incidental $(1 \mathrm{~cm})$ ICCC of the left lobe (fig. 1). Immunosuppression consisted of tacrolimus (Tac), mycophenolate mofetil (MMF) and steroids (discontinued 3 months after liver transplantation). In 2008, during hepaticojejunostomy for stenosis of biliary anastomosis, an incidental jejunal tumor was simultaneously resected. The pathohistological report revealed a $1-\mathrm{cm}$ submucosal, well-circumscribed spindle cell tumor with numerous eosinophilic collagen globules and skeinoid fibers. Mitotic figures were low, 1 mitosis per 50 high-power fields was found. Tumor necrosis and mucosal invasion were not noted. Immunohistochemically, tumor cells showed strong positivity for CD117, BCL2, $\alpha$-actin, NSE, S-100, and focally for CD34 (fig. 2). Tumor cells were desmin and cytokeratin negative. Immunohistochemical results were compatible with gastrointestinal autonomic nervous tumor, a phenotypic variant of GIST (UICC TNM classification: T1, N0, M0). The patient successfully recovered and no further treatment was initiated. Three years later, in May 2011, he developed diarrhea and reported significant weight loss $(11 \mathrm{~kg})$. White blood cell count was $7.26 \times 10^{9} / \mathrm{l}$, hemoglobin $73 \mathrm{~g} / \mathrm{l}$, and platelets $32 \times 10^{9} / \mathrm{l}$. Lactic dehydrogenase was $439 \mathrm{IU} / \mathrm{l}$ (normal range <241). Stool culture tested negative. Polymerase chain reaction for Epstein-Barr virus showed 91,200 copies/ml. Extensive diagnostic workup did not reveal recurrence of ICCC or GIST.

Bone marrow aspiration showed leukemic infiltration with dysmorphic blast populations, one resembling lymphoblasts and the other myeloblasts (fig. 3), which immunostained for CD7, partially positive for CD34 and were rarely cytochemically positive for both myeloperoxidase (MPO) and alpha-naphthyl-acetate-esterase. Cytological diagnosis was biphenotypic acute leukemia. Flow cytometry revealed expression of CD45, CD7, CD38, TdT, HLA D/DR, CD33 and marginal presence of CD34 and MPO on malignant cells. The patient's karyotype 48,XY,+21,+21[2]/46,XY[50] demonstrated AML1 (21q11) tetrasomy 21 in 1.5\% of nuclei. Molecular markers evaluated by reverse transcriptase-PCR tested negative for AML1-ETO, CBFb-MYH11, BCR-ABL p190, BCR-ABL p210 and FLT3. Due to the patient's low performance status, intensive chemotherapy was not administered. Treatment with oral agents was considered. However, due to stabile blood counts and the request of the patient it was postponed. Meanwhile, transfusional support was administered; Tac and MMF were gradually tapered with satisfactory laboratory response and no evidence of progressive disease. Two months later, the patient died at home of an unknown cause. 
Written informed consent was obtained from the patient's (adult) son for publication of this case report and any accompanying images. A copy of the written consent is available for review by the Editor-in-Chief of this journal.

\section{Conclusion}

GISTs, the most common primary mesenchymal tumors of the GIT, account for $<1 \%$ of all gastrointestinal neoplasms. This group consists of tumors of variable mesenchymal cell histogenesis that originate from the interstitial cells of Cajal by mutational activation of receptor tyrosine kinases KIT $(75-80 \%)$ or platelet-derived growth factor receptor (PDGFRA) $(<10 \%)[10]$.

The association of GISTs with leukemia has been addressed in the non-transplant population, but it has not been elucidated whether it is purely incidental or in some way pathophysiologically related. Recently, in a retrospective study on 1,892 GIST patients, Miettinen et al. [3] showed the development of myeloid leukemia in $9(0.48 \%)$ patients, with a higher incidence than that expected in the general population, suggesting an apparent nonrandom association between GIST and myeloid leukemia. The majority of leukemia cases (67\%), such as the one presented, were associated with GISTs of the small intestine. However, to date, there are no references of coexistence of GIST and biphenotypic acute leukemia in either the non-transplant or transplant population. Biphenotypic acute leukemia (BAL) is a form of rare mixed-phenotype acute leukemia that expresses both myeloid and lymphoid differentiation markers; it comprises $2-5 \%$ of all acute leukemias [11]. The prognosis of BAL is generally poor, when compared with de novo acute myeloid leukemia or acute lymphoblastic leukemia [12].

On the other hand, the coexistence of GISTs with solid tumors - synchronous or metachronous - has an incidence of up to $30 \%[1,2]$. This association is related to genetic aberrations or environmental factors predisposing the development of multi-neoplastic disease [1]. GISTs are mainly associated with luminal gastrointestinal adenocarcinomas and genitourinary neoplasms, whereas the association with primary hepatic neoplasms is rare. An extensive review of the literature yielded only 1 case of synchronous presentation of GIST and ICCC in a non-transplant patient [13]. Incidental ICCC, confirmed in the explanted liver of our patient, highlights another interesting link in the possible association of carcinogenesis of the presented multi-neoplastic disease.

Although ICCC tumorigenesis still remains unclear, the leading hypothesis supports the stepwise model by which accumulation of genetic and epigenetic alterations induced by an inflammatory process lead to the development of ICCC [14]. Pathological activation of the KIT signaling pathway, as a result of KIT or PDGFRA activating mutations, is a central event of GIST pathogenesis [10]. However, the same mutations have also been implicated in the development of acute leukemia and progression of ICCC $[15,16]$, providing a possible link between these malignancies. KIT is a receptor tyrosine kinase encoded by KIT (located at chromosome 4q12) that, together with its ligand stem cell factor, is important in hematopoiesis, gametogenesis and melanogenesis. KIT activating mutations have been described in a number of hematologic and non-hematologic malignancies, such as mastocytosis, acute leukemia, germ cell tumors and GISTs [16].

However, when discussing tumorigenesis in a transplant patient, a well-known fact is that long-term posttransplant survival carries a burden of de novo malignancy. The risk of malignancy is 2-4 times higher than in the general population [4], with the most common being skin cancer, solid tumors, posttransplantation lymphoproliferative disease, sarcomas 
and uterine cervical dysplasia [17]. Acute leukemia remains a rare complication after solid organ transplantation, with the incidence ranging from 0.12 to $2.5 \%$ [18], but with a high mortality rate (53\%) [7]. Numerous mechanisms proposed in the pathogenesis of posttransplant carcinogenesis include suppression of cytotoxic $\mathrm{T}$ cells, macrophages and natural killer cells leading to impaired immune surveillance of malignant cells, direct carcinogenic effect of immunosuppressive drugs, and oncogenic viruses. However, precise mechanisms by which they induce the occurrence of posttransplantation malignancies are still not completely understood $[17,19]$.

The unusual clinical course of this patient presenting with multi-neoplastic disease offers different, although to a great extent speculative, explanations regarding the process of tumorigenesis. Genetic predisposition has probably played an important role in the development of multi-neoplastic disease, but the contribution of immunosuppressive agents after transplantation should also not be underestimated.

\section{Disclosure Statement}

The authors declare that they have no competing interests.

\section{References}

1 Ponti G, Luppi G, Martorana D, et al: Gastrointestinal stromal tumor and other primary metachronous or synchronous neoplasms as a suspicion criterion for syndromic setting. Oncol Rep 2010;23:437-444.

-2 Sevinc A, Seker M, Bilici A, et al: Co-existence of gastrointestinal stromal tumors with other primary neoplasms. Hepatogastroenterology 2011;58:824-830.

-3 Miettinen M, Kraszewska E, Sobin L, et al: A nonrandom association between gastrointestinal stromal tumors and myeloid leukemia. Cancer 2008;112:645-649.

-4 Herrero J: De novo malignancies following liver transplantation: impact and recommendations. Liver Transpl 2009;15(suppl 2):S90-S94.

5 Saidi R, Sepehr A, Cosimi A, et al: Gastrointestinal stromal tumor in a liver transplant recipient. Transplantation 2008;85:1363.

6 Camargo MA, Boin I, Mainnardi JP, et al: Extragastrointestinal stromal tumor and liver transplantation: case report and review. Transplant Proc 2008;40:3781-3783.

7 Jiang N, Li H, Wang GS, et al: Acute leukemia, a rare but fatal complication after liver transplantation. Leuk Res 2009;33:1349-1351.

8 Wozniak L, Yang H, Lassman C, et al: Extramedullary acute myelocytic leukemia following liver transplantation for VOD with immunodeficiency. J Pediatr Gastroenterol Nutr 2011;53:346-349.

-9 Mannelli F, De Simone P, Gianfaldoni G, et al: Atypical acute leukemia early after liver transplantation. Transplant Proc 2009;41:3945-3946.

10 Miettinen M, Lasota J: Gastrointestinal stromal tumors: review on morphology, molecular pathology, prognosis, and differential diagnosis. Arch Pathol Lab Med 2006;130:1466-1478.

11 Borowitz M, Bene M, Harris N, et al: Acute leukemias of ambiguous lineage; in Swerdlow S, Campo E, Harris N, Jaffe E, Pileri S, Stein H, Thiele J, Vardiman J (eds): WHO Classification of Tumours of Haematopoietic and Lymphoid Tissues. Lyon, IARC Press, 2008, pp 150-155.

12 Legrand O, Perrot JY, Simonin G, et al: Adult biphenotypic acute leukaemia: an entity with poor prognosis which is related to unfavourable cytogenetics and P-glycoprotein over-expression. Br J Haematol 1998;100:147-155.

13 Lao XM, Ye ZY, Guo RP, et al: A gastrointestinal stromal tumor of the jejunum associated with intrahepatic cholangiocarcinoma and pulmonary hamartoma: a case report. Acta Oncol 2009;48:934-937.

14 Kumar M, Zhao X, Wang X: Molecular carcinogenesis of hepatocellular carcinoma and intrahepatic cholangiocarcinoma: one step closer to personalized medicine? Cell Biosci 2011;24:5.

15 Mansuroglu T, Ramadori P, Dudás J, et al: Expression of stem cell factor and its receptor c-Kit during the development of intrahepatic cholangiocarcinoma. Lab Invest 2009;89:562-674.

16 Miettinen M, Lasota J: KIT (CD117): a review on expression in normal and neoplastic tissues, and mutations and their clinicopathologic correlation. Appl Immunohistochem Mol Morphol 2005;13:205-220.

17 Benlloch S, Berenguer M, Prieto M, et al: De novo internal neoplasms after liver transplantation: increased risk and aggressive behavior in recent years? Am J Transplant 2004;4:596-604. 


\section{Case Reports in Oncology}

\begin{tabular}{l|l}
\hline \multicolumn{2}{l}{ Case Rep Oncol 2013;6:163-168 } \\
\hline DOI: $\underline{10.1159 / 000348817}$ & $\begin{array}{l}\text { ○ 2013 S. Karger AG, Basel } \\
\text { www.karger.com/cro }\end{array}$ \\
\hline
\end{tabular}

Mrzljak et al:: Metachronous Gastrointestinal Stromal Tumor and Acute Leukemia after Liver Transplantation for Cholangiocellular Carcinoma: Is There a Link?

18 Cho YU, Chi HS, Park CJ, et al: Two cases of post-liver transplant acute myeloid leukemia in Korean adults: review of bibliographies and comparison with post-renal transplant acute myeloid leukemia. Ann Hematol 2008;87:513-514.

19 Penn I: Why do immunosuppressed patients develop cancer? Crit Rev Oncog 1989;1:27-52.
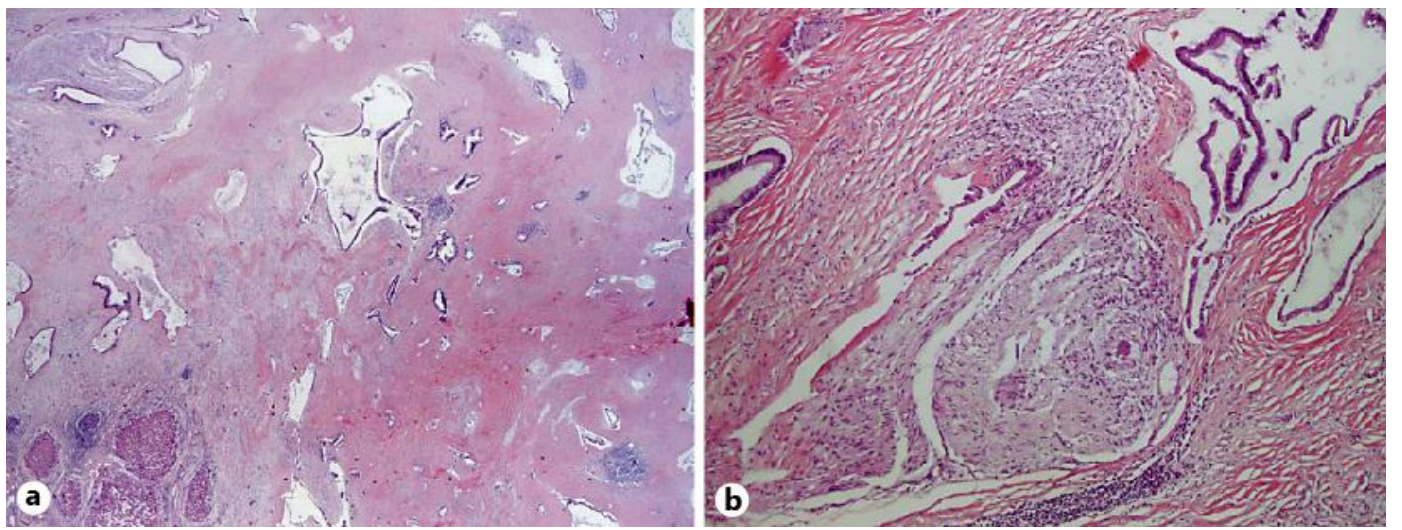

Fig. 1. ICCC. a Tumor showed glandular appearance with prominent desmoplastic stroma. HE. $\times 2$. b Perineural invasion of ICCC. KS-Giemsa. $\times 20$.

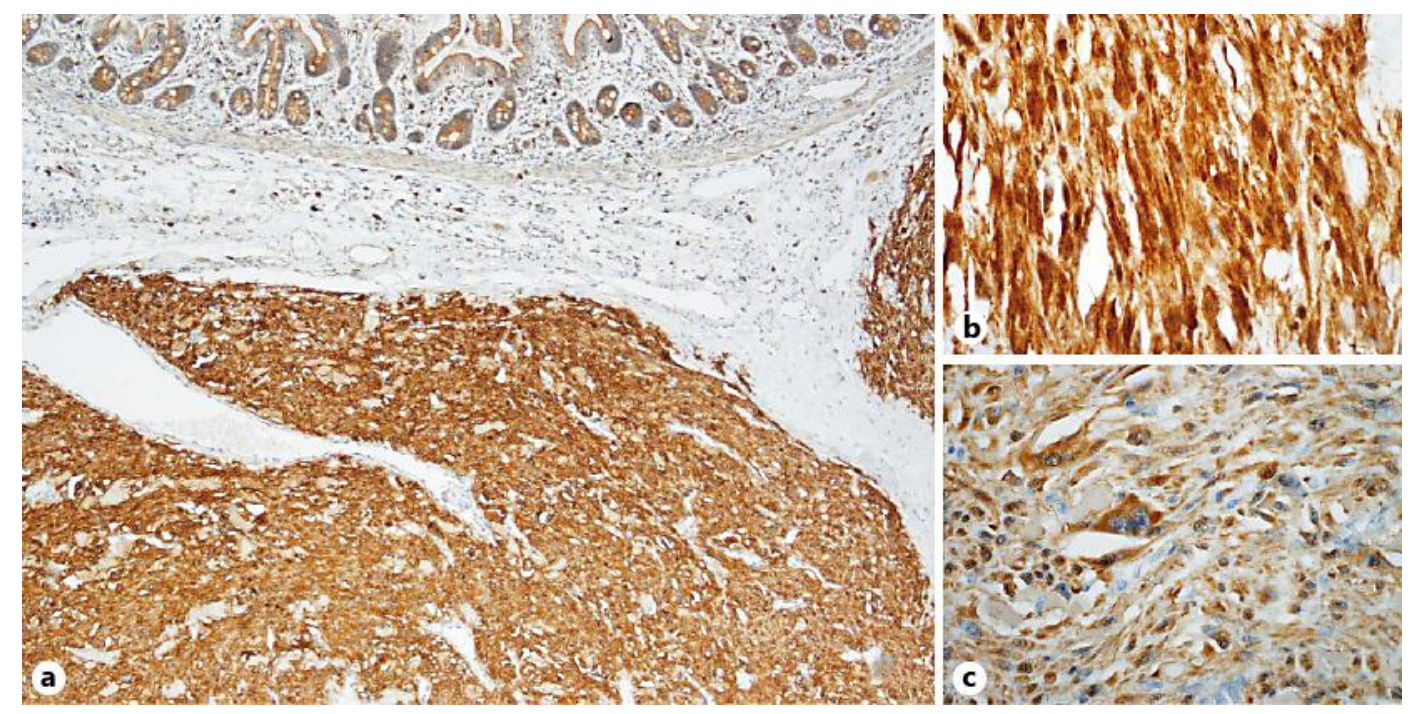

Fig. 2. Immunohistochemical staining of the GIST. a CD117 staining. $\times 2$. b NSE staining. $\times 20$. c S-100 staining. $\times 20$. 


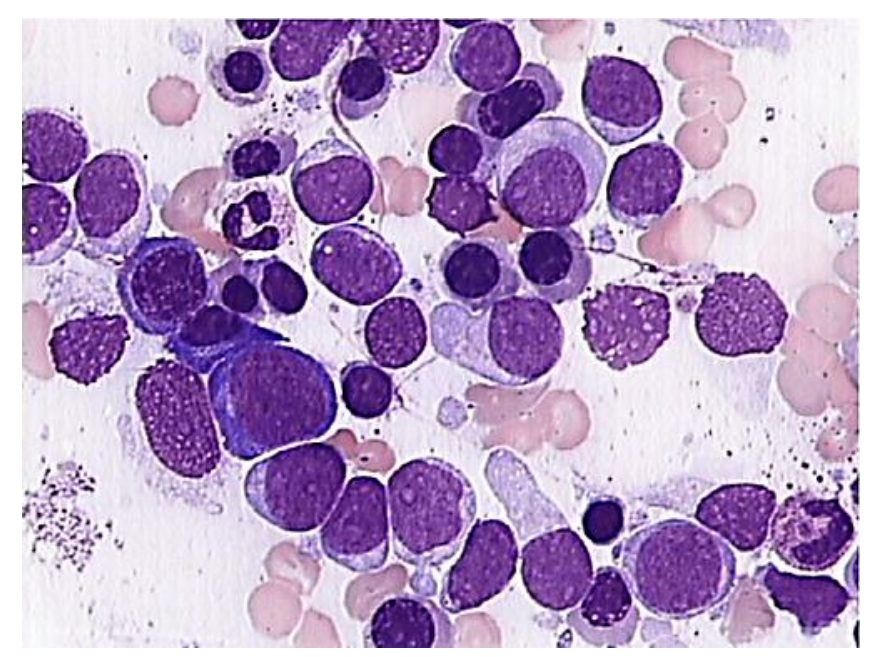

Fig. 3. Bone marrow aspirate. Biomorphic blast population, one resembling lymphoblasts and the other myeloblasts. May-Grünwald Giemsa. ×1,000. 